\title{
Sonographic detection of the lead point in intussusception
}

\author{
M D Stringer, S N J Capps, S M Pablot
}

\begin{abstract}
Using ultrasonography, a caecal duplication cyst was identified as the pathological lead point in a 6 year old boy with acute intussusception. The patient underwent definitive surgery rather than inappropriate treatment by attempted radiological reduction. Various pathological lead points in intussusception can now be defined by ultrasound.
\end{abstract}

Most cases of childhood intussusception are secondary to lymphoid hyperplasia in the terminal ileum but in as many as $10 \%$ of patients a more definite pathological lead point, such as a Meckel's diverticulum, small bowel tumour, or duplication cyst, is responsible. ${ }^{1}$ Such patients are at risk of recurrent intussusception and other complications of the underlying pathology and therefore should be treated surgically rather than by radiological reduction methods. In recent years ultrasonography has emerged as a useful diagnostic aid in intussusception. We believe that the role of ultrasound extends beyond that of confirming the diagnosis. It can also be used in some cases to identify pathological lead points, thus enabling simpler and safer management of the patient. This is exemplified by the following unusual case report.

\section{Case report}

A previously well 6 year old boy was admitted with a two day history of colicky abdominal pain and bile stained vomiting. On examination he appeared well without signs of dehydration
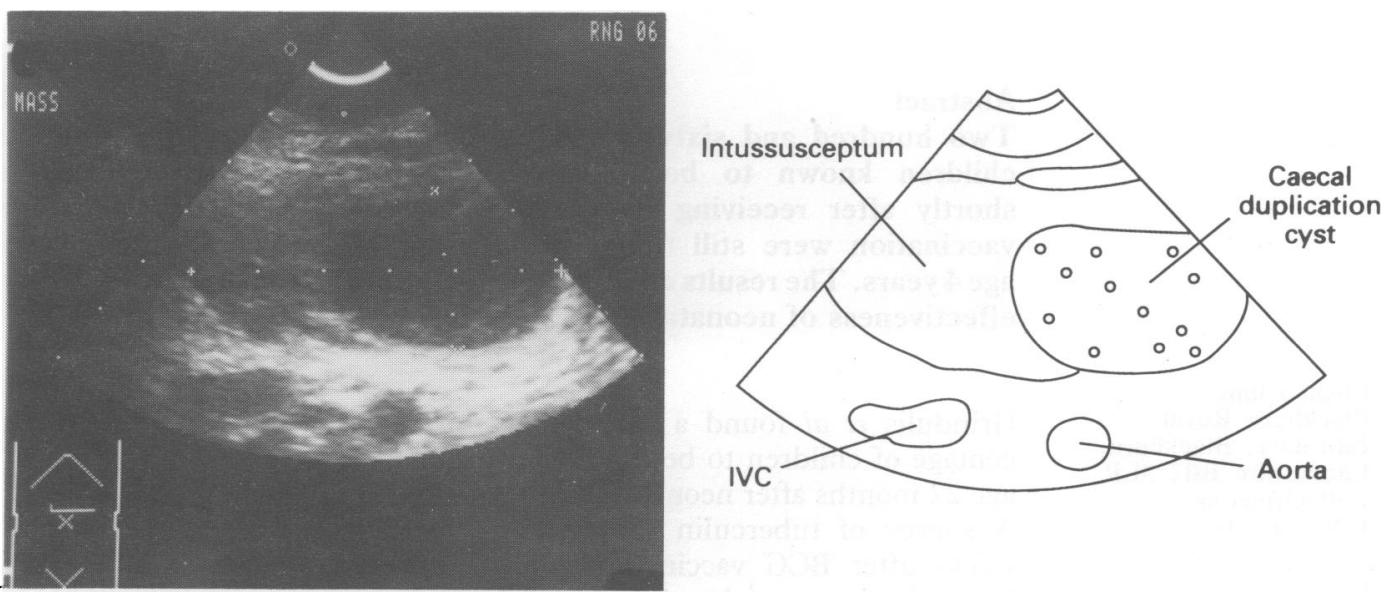

\section{Discussion}

Ultrasonographic identification of a pathological lead point in this patient avoided an inappropriate attempt at radiological reduction. If such an attempt had succeeded, the child would have been exposed to the risks of recurrent intussusception and other potential complications of the duplication cyst. The cyst is unlikely to have been detected during a pneumatic reduction judging by the reported experience of similar lesions with barium enemas, which often provide even better radiological definition.

We believe that ultrasonography is underused in the management of childhood intussusception. During the past two years we have used both ultrasonography and contrast enemas to confirm
Queen Mary's Hospital for Children,

Carshalton, Surrey, Department of Paediatric Surgery

$M$ D Stringer

$S$ N J Capps

Department of Radiology S M Pablot

Correspondence to: Mr M D Stringer, Department of Paediatric Surgery Institute of Child Health 30 Guilford Street, London WCIN IEH.

Accepted 21 November 1991

Figure 1 Longitudinal section of cystic lead point at the apex of the intussusceptum in the distal transverse colon (5 MHz (Arch Dis Child 1992;67:529-30) sector probe); IVC=inferior vena cava. 


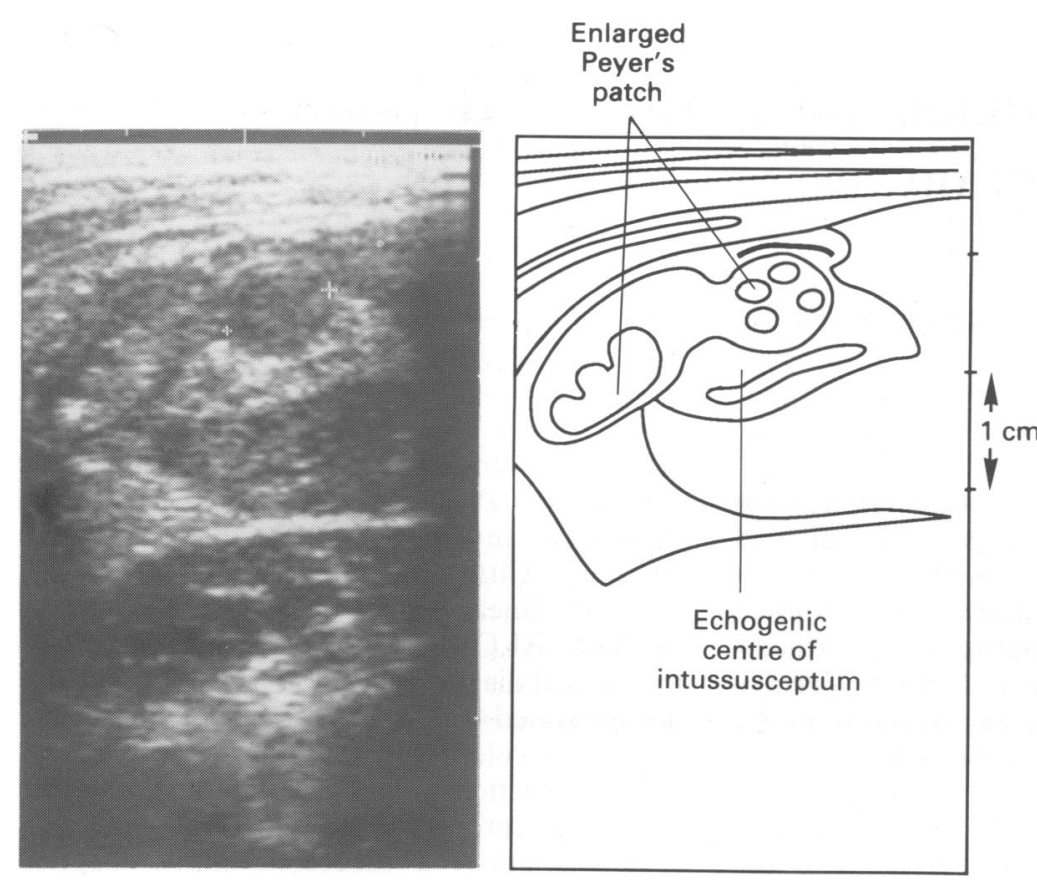

Figure 2 Typical discrete lesion (enlarged Peyer's patch) seen in transverse section at the apex of an 'idiopathic' ileocolic intussusception ( $7.5 \mathrm{MHz}$ linear view).

or exclude intussusception in children not obviously requiring surgery, and our experience accords well with that of Pracros et al who found ultrasonography safe and reliable with neither false positives nor negatives. ${ }^{2}$ However, clearly the experience of the sonographer is important and if the diagnosis of intussusception remains uncertain after scanning with ultrasound, contrast studies should be performed.
There are several reports of children with other pathological lead points detected by ultrasound, including Meckel's diverticula ${ }^{3}$ and a small bowel lymphoma. ${ }^{4}$ One child with a false cystic lead point due to encysted fluid within the intussusception has been described but the appearances differ from our case. ${ }^{5}$ In some patients we consider that it has been possible to identify the lymphoid hyperplasia of 'idiopathic' intussusception within the intussusceptum (fig 2). Yet another diagnostic role of ultrasound is in the detection of small bowel intussusception which often proves difficult with conventional radiology. Reported examples include ileoileal intussusception occurring postoperatively ${ }^{4}$ and in Henoch-Schönlein purpura. ${ }^{6}$

In summary, not only is ultrasonography able to accurately diagnose intussusception, including the more difficult small bowel intussusception, but it may also be used to define a pathological lead point as demonstrated by this case report. This contributes to safer and simpler patient management.

1 Ong NT, Beasley SW. The leadpoint in intussusception. F Pediatr Surg 1990;25:640-3.

2 Pracros JP, Tran-Minh VA, Wright C. Ultrasound in diagnosis of intussusception. Lancet 1985;ii:733-4.

3 Itagaki A, Uchida M, Ueki K, Kajii T. Double targets sign in ultrasonic diagnosis of intussuscepted Meckel's diverticulum. Pediatr Radiol 1991;21:148-9.

4 Dinkel E, Dittrich M, Pistor G, Weitzel D, Greinacher I. Sonographic diagnosis of intussusception in childhood. $Z$ Kinderchir 1983;38:220-3.

5 Kenney IJ. Ultrasound in intussusception: a false cystic lead point. Pediatr Radiol 1990;20:349-50.

6 Martinez-Frontanilla LA, Silverman L, Meagher DP. Intussusception in Henoch-Schönlein purpura: diagnosis with ultrasound. $\mathcal{F}$ Pediatr Surg 1988;23:375-6.

\title{
Tuberculin skin reactivity four years after neonatal BCG vaccination
}

\author{
L P Ormerod, J M Garnett
}

Chest Clinic,

Blackburn Royal

Infirmary, Blackburn,

Lancashire BB2 3LR

L P Ormerod

J M Garnett

Correspondence to:

Dr Ormerod.

Accepted 29 July 1991

\begin{abstract}
Two hundred and sixty one of 279 (93.5\%) children known to be tuberculin positive shortly after receiving their neonatal BCG vaccination were still tuberculin positive at age 4 years. The results confirm the continuing effectiveness of neonatal BCG at 4 years.
\end{abstract}

Grindulis et al found a surprisingly low percentage of children to be tuberculin positive at age 22 months after neonatal BCG vaccination. ${ }^{1}$ A survey of tuberculin reactivity six to nine weeks after BCG vaccination confirmed very high effectiveness. ${ }^{2}$ A proportion of the children from that survey had their tuberculin reactivity (Arch Dis Child 1992;67:530-1) retested at age 4 years.

\section{Subjects and methods}

Altogether 863 infants whose ethnic origin was the Indian subcontinent and who were born between August 1984 and July 1985 were tuberculin tested six and nine weeks after neonatal vaccination. ${ }^{2}$ A total of $846(97 \cdot 3 \%)$ were positive grades 1 or 2 . A random one third of tuberculin positive infants from each month (totalling 279) had their skin tuberculin test (tine test, Lederle) repeated at the age of 4 years.

\section{Results}

Of the 279 children who had grade 1 or 2 positive tuberculin tests in $1984 / 5$ tested, 18 $(6 \cdot 5 \%)$ were tuberculin negative and 261 (93.5\%) 